\title{
A Case of CONGENITAL ATRESIA OF THE JEJUNUM (with Recovery) \\ BY
}

G. BRUTON SWEET, M.B., Ch.M.

Pediatrist, Auckland Hospital, N.Z.

AND

CARRICK ROBERTSON, M.B., B.S., F.R.C.S.

Surgeon, Auckland Hospital, N.Z.

Of the various forms of congenital malformation few, if any, have been, up to the present time, so uniformly fatal as congenital atresia of the small intestine. From the nature of this condition with complete blocking or loss of continuity of the alimentary canal, it is obvious that an operation affords the only hope of life, and a study of recorded cases shows that only a very few infants have survived.

Davis and Poynter ( ${ }^{1}$ ) collected 392 cases on congenital occlusion of the intestine between the pylorus and the rectum. Of these, 194 were examples of a single atresia of the duodenum or jejunum, and 67 were cases of multiple atresia, slightly more than fifty per cent. of the latter consisting of from two to nine occlusions of the jejunum and upper ileum. None of the cases of this series recovered; in one of atresia of the jejunum external enterostomy was performed and the infant died six hours later.

Sheldon $\left({ }^{2}\right)$ collected 28 cases from the record of autopsies performed at the Hospital for Sick Children, Great Ormond Street, London, since the commencement of the present century, but no mention is made of any attempt by surgery to avert the fatal issue.

The first attempt at cure by operation was made by Bland Sutton. The first case in which operation was performed successfully was reported by Fockens ( ${ }^{3}$ ) who made an anastomosis between the segments. The second successful case was reported by Ernst $\left({ }^{4}\right)$ who brought the ileum up anterior to the colon and anastomosed it to the duodenum. The third case is reported by Richter. ${ }^{5}$ ) of Chicago, who performed posterior gastro-enterostomy.

As far as we are able to discover the case here reported is the fourth successful case on record, and we are publishing it in the hope that others will soon follow, for with early diagnosis and surgical intervention, we consider that the prognosis of congenital atresia when single and situated in the upper small intestine is by no means hopeless and that a fair proportion of cases should recover. 
The degree of vitality displayed by the new-born is occasionally surprising, and one of us has seen an instance of congenital atresia of the small intestine in which the infant survived for more than three weeks, though no food was retained by the stomach after birth and no attempt was made to prolong life by means of subcutaneous injections or other means.

A female infant, nine days old, was seen by one of us in consultation with Dr. Gladys Montgomery on September 29th, 1926, for persistent vomiting. Nothing abnormal had been noted about the baby for the first three days of life except that very little meconium was passed. On one occasion only a small amount of brownish fluid had been passed. Vomiting commenced as soon as lactation was established; it had persisted ever since and was projectile in character. Occasionally a feed was retained, but this was seldom, and it was noticed that after the next feed a larger amount was ejected. The baby, which had weighed $7 \mathrm{lbs}$. at birth, now weighed only $5 \frac{1}{2}$ lbs.

On examination it was found to be feeble, wasted, with loose folds of skin over abdomen and limbs, subnormal temperature, and sunken fontanelle. A feed was given and promptly and forcibly ejected; the vomited fluid being deeply stained with bile. Gastric peristalsis was visible in short waves which stopped abruptly at the mid-line of the epigastrium.

A diagnosis of congenital atresia of the duodenum was made and it was decided to give the baby the benefit of the very slender chance that operation offered for recovery. It was admitted to the Auckland Hospital on the same evening and on the following morning, after preparation with subcutaneous salines and administrations of saline and glucose by the rectum, laparotomy was performed.

The stomach and duodenum were found to be much dilated, the latter ending in a little bulge behind the peritoneum over the lumbar spine. There was no cord or other vestigial structure connecting this mass with the proximal end of the jejunum. The commencement of the jejunum could not be distinguished, for the end of the uppermost loop apparently disappeared into the extra-peritoneal space. The coils of collapsed small intestine all lay in the right upper quadrant of the abdomen; there appeared to be fewer coils than usual, and they overlaid the ascending colon and cæcum. The large bowel was normal in appearance, but there was no great omentum.

As the child was nearly moribund it was not thought advisable to join the extraperitoneal knob of duodenum to the jejunum lying some distance away, for the quickest operation that could be done was anterior gastrojejunostomy. Immediately this was finished the distension of the stomach and duodenum disappeared by the filling up of the coils of small intestine.

For the next three days tine condition of the infant was critical, the vomiting persisted to some extent, and often large quantities of bile-stained fluid were returned. However, by keeping the infant in a sitting position, and washing out the stomach with a $5 \%$ solution of Sodium Bicarbonate, improvement took place and some of the feeds were retained.

Breast feeding was resumed on the sixth day after operation, and the baby was sent home to be nursed on October 9th. At that date vomiting of clear, bile-stained fluid occurred two or three times a day; the motion was normal, and the baby had gained in strength, and weighed 5 lbs. 10 ozs.

Twelve days later, as vomiting still continued, and the baby had lost a few ounces in weight, she was readmitted for a second operation.

It was obvious that a great deal of the secretions of the liver and pancreas, which had to pass into the stomach on the way to the intestines, were lost by vomiting, and it was therefore deoided to make a short circuit between the duodenum and the jejunum.

This, which proved to be a somewhat difficult operation on such a tiny infant, was accordingly done on October 22nd. The anterior aspect of the second part of the duodenum was joined to the jejunal loop distal to the previous anastomosis. 


\section{CONGENITAL ATRESIA OF THE JEJUNUM}

Vomiting occurred shortly after operation and the baby became extremely dehydrated, and too feeble even to suck.

Blood transfusion into the longitudinal sinus was performed and saline was given subcutaneously. On the third day after operation, when apparently moribund, gastric lavage with sodium bicarbonate solution $(5 \%)$ was successful in arresting vomiting, and thereafter recovery was uninterrupted.

On the sixth day breast feeding was resumed and the baby was discharged from Hospital on November 1lth, when she weighed 4 lbs. 14 ozs., to report as an out-patient. On November 16th she appeared quite well; weight 5 lbs. 10 ozs.

On December 7th her weight was 6 lbs. 12 ozs., and on December 29th she was quite well and weighed $8 \mathrm{lbs} .2 \mathrm{ozs}$. There had been no vomiting for some weeks except that occasionally a small quantity of yellow fluid was returned. On January 30th, the weight was 9 lbs. 12 ozs. and on February 13th 10 lbs. 12 ozs.

The baby was now in excellent health, bright and intelligent.

\section{REFERENCES.}

1. Davis and Poynter, J. of Surg., Gynecol. and Obst., 1922, 34.

2. Sheldon, Arch. of Dis. in Child., 1926, I, 279.

3. Fockens, Nedarb. Tijdschr. V. Genesk., 1911, XVI, 655.

4. Ernst, Brit. Med. J., 1916, i., 644.

5. Richter, Abt's Pediatrics, III, 512. 